\section{Risk of Invasive Fungal Infec- tions and Osteonecrosis of Jaw in Patients Exposed to Current Therapeutic Drugs for COVID-19}

Sir,

Recently appreciable work has been done by the authors involved in a study carried out in Peshawar, Pakistan, to analyse the role of tocilizumab in combination with the standard treatment for COVID-19. I would like to bring attention of authors towards the role of tocilizumab and corticosteroids in invasive fungal infections of maxillofacial region and osteonecrosis of jaws.

As we know, mild COVID can be managed by symptomatic and supportive treatment with antipyretics, analgesics, and oxygen therapy; whereas, for the treatment of progressive disease, various therapeutic agents including tocilizumab and corticosteroids have been proposed. ${ }^{2}$ Tocilizumab is humanised monoclonal antibody that binds to interleukin- 6 (IL-6) receptors, thus inhibiting the IL-6-mediated inflammatory reaction. The corticosteroids, including dexamethasone and methylprednisolone, are used due to their anti-inflammatory action. Previously in another study, it was shown that both these drugs had shown improved outcomes in patients with COVID-19 pneumonia. $^{3}$

Recently, during second wave of COVID-19 in India, a surge in cases of invasive fungal infections, particularly rhino-cerebro-orbital form of mucormycosis and aspergillosis, has been noted. ${ }^{4}$ The causative agent of rhino-cerebro-orbital type of mucormycosis is a saprophytic fungus of the family mucoraceae. Its spores reside in paranasal sinuses of the host and cause vascular invasion and thrombosis, ultimately resulting in widespread tissue necrosis. Immunocompromised patients, diabetics, and patients taking steroids are more susceptible to these infections. A recent retrospective study performed in India showed that 16 out of 18 COVID-19 positive patients with rhino-cerebro-orbital mucormycosis were treated with steroids, while 15 of them had uncontrolled diabetes. ${ }^{4}$ Another case of rhino-cerebro-orbital mucormycosis was reported from Brazil, which is believed to be caused by poorly controlled diabetes and concomitant administration of corticosteroids. ${ }^{5}$ Similarly, risk of maxillofacial aspergillosis, another invasive fungal infection, which is also seen in immunocompromised patients, should not be underestimated.

Due to anti-inflammatory and immunomodulatory action of both corticosteroids and tocilizumab, it can be assumed that the combination therapy with both these drugs could result in the development of fungal infection or exacerbation of pre-existing fungal disease in predisposed patients. Therefore, physicians should be careful while prescribing steroids and tocilizumab for treatment of COVID-19 pneumonia, particularly in patients having uncontrolled diabetes mellitus. These fungal infections are quite invasive in nature and their intra-orbital and intra-cranial extension is associated with high morbidity and mortality. Hence, regular follow-up should be planned in these COVID patients, not only to monitor glycemic control but also to detect early signs of invasive fungal infections.

Medication-related osteonecrosis of jaw (MRONJ) is a condition characterised by necrosis of jaw bones in patients exposed to a range of drugs, including bisphosphonates, antibody to RANKL (Receptoractivatorfornuclearfactorkappa-beta ligand), angiogenic inhibitors, tyrosine kinase inhibitors and vascular endothelial growth factors (VEGF) inhibitors. ${ }^{6}$ It is also known that concomitant treatment with steroids and systemic conditions like diabetes and compromised immune system can exacerbate the osteonecrosis of jaw. Although tocilizumab has not been included in drugs that lead to MRONJ, a case has been reported in 2018 describing a patient, treated with tocilizumab for rheumatoid arthritis, who developed features of MRONJ without history of use of bisphosphonates or other related drugs. $^{7}$

Many old-age patients are usually exposed to various drugs associated with MRONJ for the treatment of osteoporosis or other conditions. As elderly patients are more likely to develop progressive COVID pneumonia and severe disease requiring aggressive medical treatment with drugs including corticosteroids and tocilizumab, thus making them more susceptible to develop osteonecrosis ofjaw. ${ }^{8}$

Therefore, we can conclude that the regimens, comprising of corticosteroids and tocilizumab, used eitheralone or in combination for the treatment of progressive COVID pneumonia, should be used with caution and medical community, should be aware of the associated potential risks of invasive fungal infections of maxillofacial region and osteonecrosis of jaw. Diabetic patients and those taking any of the drugs associated with MRONJ are more at risk for developing these conditions.

Further studies are needed to explain the possible role of COVID-19 and the drugs used for the treatment of COVID-19 pneumonia towards risk of acquiring invasive fungal infection of the maxillofacial region and osteonecrosis of jaw.

\section{CONFLICT OF INTEREST:}

The author declared no conflict of interest.

\section{AUTHOR'S CONTRIBUTION:}

MAA: Concept, literature review, manuscript writing, and proofreading.

\section{REFERENCES}

1. Amin S, Rahim F, Bahadur S, Noor M, Mahmood A. The effect of Tocilizumab on inflammatory markers in survivors and non survivors of severe COVID 19. J Coll Physicians 
Surg Pak 2021; 30(1): S7-S10. doi: 10.29271/jcpsp. 2021.01.57.

2. Majumder J, Minko T. Recent developments on therapeutic and diagnostic approaches for COVID-19. AAPS J 2021; 23(1):14. doi: 10.1208/s12248-020-00532-2.

3. Mikulska M, Nicolini LA, Signori A, Di Biagio A, Sepulcri C, Russo $C$, et al. Tocilizumab and steroid treatment in patients with COVID-19 pneumonia. PLOS ONE 2020; 15(8):e0237831. doi: 10.1371/journal.pone.0237831.

4. Moorthy A, Gaikwad R, Krishna S, Hegde R, Tripathi KK, G Kale $P$, et al. SARS-CoV-2, uncontrolled diabetes and corticosteroids - an unholy trinity in invasive fungal infections of the maxillofacial region? A retrospective, multi-centric analysis. J Maxillofac Oral Surg 2021; 1-8. doi: 10.1007/s12663-021-01532-1.

5. Pauli AM, Pereira LM, Monterio LM, de Camargo AR, Rabelo GD. Painful palatal lesion in a patient with COVID-19. Oral Surg Oral Med Oral Pathol Oral Radiol 2021; 131(6):620-5. doi: 10.1016/j.0000.2021.03.010.

6. Wan JT, Sheeley DM, Somerman MJ, Lee JS. Mitigating osteonecrosis of the jaw (ONJ) through preventive dental care and understanding of risk factors. Bone Res 2020; 8:14. doi: 10.1038/s41413-020-0088-1.

7. Bindakhil MA, Mupparapu M. Osteomyelitis of the mandible exhibiting features of medication -related osteonecrosis in a patient with history of tocilizumab treatment. J Orofacial Sci 2018; 10(1):53. Doi: 10.4103/jofs.jofs_46_18

8. Wu C, Chen X, Cai Y, an Xia J, Zhou X, Xu S, et al. Risk factors associated with acute respiratory distress syndrome and death in patients with coronavirus disease 2019 pneumonia in Wuhan, China. JAMA Intern Med; 180(7): 934-3. doi: 10.1001/jamainternmed.2020.0994.

Muhammad Adil Asim

Department of Oral and Maxillofacial Surgery, Shifa College of Dentistry, Shifa Tameer-e- Millat University, Islamabad,

Pakistan

Correspondence to: Dr. Muhammad Adil Asim, Department of Oral and Maxillofacial Surgery, Shifa College of Dentistry, Shifa Tameer-e-Millat University, Islamabad, Pakistan

E-mail: adil_asim@yahoo.com

Received: May 26, 2021; Revised: June 30, 2021; Accepted: July 03, 2021

DOI: https://doi.org/10.29271/jcpsp.2021.JCPSPCR.CR150 